\title{
Workplace health promotion for older workers: a systematic literature review
}

\author{
Andrea Poscia ${ }^{1 *}$, Umberto Moscato ${ }^{1}$, Daniele Ignazio La Milia ${ }^{1}$, Sonja Milovanovic ${ }^{1}$, Jovana Stojanovic ${ }^{1}$, \\ Alice Borghini ${ }^{1}$, Agnese Collamati ${ }^{2}$, Walter Ricciardi ${ }^{1,3}$ and Nicola Magnavita ${ }^{1}$
}

\begin{abstract}
Background: Aging of the workforce is a growing problem. As workers age, their physical, physiological and psychosocial capabilities change. Keeping older workers healthy and productive is a key goal of European labor policy and health promotion is a key to achieve this result. Previous studies about workplace health promotion (WHP) programs are usually focused on the entire workforce or to a specific topic. Within the framework of the EU-CHAFEA ProHealth65+ project, this paper aims to systematically review the literature on WHP interventions specifically targeted to older workers (OWs).

Methods: This systematic review was conducted by making a comprehensive search of MEDLINE, ISI Web of Science, SCOPUS, The Cochrane Library, CINAHL and PsychINFO databases. Search terms included ageing (and synonyms), worker (and synonyms), intervention (and synonyms), and health (and synonyms). The search was limited to papers in English or Italian published between January, $1^{\text {st }} 2000$ and May, $31^{\text {st }} 2015$. Relevant references in the selected articles were also analyzed.

Results: Of the 299 articles initially identified as relating to the topic, 18 articles met the inclusion criteria. The type, methods and outcome of interventions in the WHP programs retrieved were heterogenous, as was the definition of the age at which a worker is considered to be 'older'. Most of the available studies had been conducted on small samples for a limited period of time.

Conclusion: Our review shows that, although this issue is of great importance, studies addressing WHP actions for OWs are few and generally of poor quality. Current evidence fails to show that WHP programs improve the work ability, productivity or job retention of older workers. In addition, there is limited evidence that WHP programs are effective in improving lifestyles and concur to maintain the health and well-being of older workers. There is a need for future WHP programs to be well-designed so that the effectiveness and cost-benefit of workplace interventions can be properly investigated.
\end{abstract}

Keywords: Workplace health promotion, Aging workforce, Active ageing, Occupational health, Public health, Lifelong learning, Lifestyle, Frailty, Ageism, Labor policy

\section{Background}

In the last 50 years, many industrialised countries have witnessed a change in the age structure of the population [1]. The International Labor Organization has estimated that by 2025 , there will be a $32 \%$ increase in the number of people aged over 55 years. Older people will make up approximately $30 \%$ of the population in Europe and

\footnotetext{
* Correspondence: andrea.poscia@unicatt.it

${ }^{1}$ Department of Public Health, Università Cattolica del Sacro Cuore, Rome, Italy

Full list of author information is available at the end of the article
}

North America, $21 \%$ in Asia and $17 \%$ in Latin America. Thus, in Europe, the current demographic trend is towards an increasingly aged workforce [2].

There are several reasons for this phenomenon: first of all the baby boom that followed the Second World War and the low birth rates of the 1980s [3], which together have led to a decline in the inflow of young workers. The increase in life expectancy and the lack of social resources has led most European Governments to raise the retirement age. Additionally, the increased number of older employees in the workforce is due 
mainly to workers delaying retirement, training for alternative careers and prolonging their longevity thanks to advances in healthcare [4].

The definition of older worker (OW) is still controversial, since different agencies and organizations are using a broad spectrum of ages, ranging from 40 to 65 years, or more. For example, the U.S. Department of Labor, in agreement with most of the literature [5], considers workers to be older if aged 55 years or more [6], whereas the US Age Discrimination in Employment Act [7] provides protection for anyone in the workplace over the age of 40 years. In the workplace sector, workers aged over 45 years are generally considered to be "older" [3].

OWs may differ from their younger counterparts due to a number of physical/biological, psychological/mental, and social characteristics that influence their needs, expectations and challenges [8]. Physical functions, including sensory abilities (e.g. eyesight and hearing), muscular function (e.g. balance, strength and flexibility), aerobic capacity (e.g. $\mathrm{VO}_{2} \max$ ), reaction time and speed, immune response and the ability to maintain homeostasis all decline with age, and this deterioration becomes even more pronounced after the age of 50 [9]. In addition, OWs usually have a higher prevalence of aging-related metabolic disorders such as abdominal obesity, hypertension, hyperglycemia, and dyslipidemia [10]. Consequently, they are often subjected to polypharmacy, with lower quality of life, reduced mobility and mild cognitive impairment, all of which are of concern at the workplace $[11,12]$. Bearing in mind that OWs might become more sensitive to changes and less willing to accept them, it is highly probable that the majority will manifest difficulty in adjusting to new job techniques and conditions at the workplace. Moreover, published reports show an increase in perceived age discrimination among OWs which often results in negative feelings, such as uselessness, powerlessness and low self-esteem [13]. Because the ageing workforce has become an increasingly important occupational health issue, especially in relation to a nation's economic prosperity, maintaining a healthy and productive workforce is a key goal of European labor policy [14].

The worksite is generally a promising setting for health promotion. Theoretically, in the workplace, where employees spend a great amount of time, a number of promotion strategies can be used to provide opportunities for behavioral changes. Furthermore, the occupational health $(\mathrm{OH})$ service, which is part of the work organization, can use its know-how to introduce effective workplace health promotion (WHP) interventions [15], encouraging employees to take own responsibility and stimulating self-help, through participatory ergonomics [16].
Although there has been extensive investigation of WHP programs, these studies, however, are usually focused on the entire workforce $[17,18]$ and to a specific topic, such as healthy eating [16], or presenteeism [17]. Review studies on OWs, on the other hand, are specifically targeted to a single topic, such as safety and health needs of OWs [19], or OH services in the workplace, [20]. A recent review on the effectiveness of WHP for OWs found only 4 studies on early retirement work ability and productivity [21]. Our aim was to determine whether a wider range of WHP interventions developed specifically for older adults could significantly improve their ability to remain in the workplace and adapt to modern work methods and health conditions.

Although Pitt-Catsouphes et al. [22] claim that the official classification of promotion programs is still inadequate, there appear to be three broad categories: screening activities to identify potential health risks (e.g. ergonomic assessments; health risk assessments, etc.); lifestyle management activities to improve health and to prevent/minimize health risks, including those associated with chronic conditions (e.g. exercise programs; healthy food options in cafeterias, etc.); and onthe-job lifelong-learning interventions that encourage employees to remain in the workforce. Promotion programs should also focus on developing internal policies for OWs so as to address disparities among subpopulations of workers and thus avoid ageism [23, 24].

When 'healthy ageing management' is concerned, $\mathrm{OH}$ services could probably play a key role in the workplace. One study suggested that policies should look at ageing workers rather than older workers, starting with those as young as 45 . It also stated that $\mathrm{OH}$ strategies are needed to address the challenges of an ageing workforce, which include the prevention of work-related diseases, diminished work performance due to chronic diseases and the promotion of health and workability [20,25]. Organizations, both large and small, can engage in this new strategy by systematically integrating their health promotion safety and environmental programs and policies [16, 26]. Furthermore, workplace ergonomics should be age-appropriate and bear in mind the capabilities and limitations of older employees [16]. These concepts are reasonable, but there is still no sufficient evidence that WHP programs for OWs based on these assumptions are attractive to workers and companies.

The purpose of this review was to systematically summarize and scientifically appraise the literature on WHP for OWs, identify the institutions and study groups active in this field and the type of activities performed. This "state of the art" picture might help recognizing and spreading good practices in workplaces. 


\section{Methods}

\section{Search strategy}

Comprehensive database searches were performed within the framework of the EU-CHAFEA ProHealth65+ project by two independent investigators to identify potentially relevant articles from MEDLINE, ISI Web of science, SCOPUS, The Cochrane Library, CINAHL and PsychINFO databases. The search strategy, based on the implemented PICO model, was developed first devised for use in MEDLINE and subsequently adapted for the other databases. The combination of keywords used at MEDLINE were: (aging OR ageing OR elderly $O R$ aged OR old) AND (worker* OR workforce OR employee* OR farmer* OR craftsman OR laborer) AND (Health OR safety) AND (promote* OR prevent* OR intervention OR program*) AND workplace AND (Active Ageing and Job OR Well-Being OR work ability OR disability $O R$ impairment $O R$ rehabilitation $O R$ fitness $O R$ capacity $O R$ retention $O R$ re-entry $O R$ employability $O R$ adaptation OR satisfaction $O R$ attitude* OR discrimination $O R$ integration $O R$ productivity $O R$ absenteeism $O R$ presenteeism OR mental health OR stress OR learning $O R$ life style* OR occupational disease*).

\section{Inclusion and exclusion criteria}

Studies were considered eligible if they reported interventions which were conducted specifically on OWs, or at least had the aim to promote healthy/active ageing in the working population. If the intervention was targeted at both the entire workforce and younger workers, only subgroup analyses for ageing workers were deemed eligible. The studies included had to be original research (randomized controlled trials (RCT), quasi-experimental studies (NCT), pre-post, cohort, case-control, ecological and cross-sectional studies), or primary studies derived from relevant systematic reviews and meta-analyses.

Studies that analyzed the clinical course of age and/or work-related illnesses were excluded. The search was limited to papers in English and Italian published between January, $1^{\text {st }} 2000$ and May, $31^{\text {st }} 2015$.

\section{Study selection}

After completion of the searches and exclusion of duplicate studies, the initial screening of publications included an independent review of titles and abstracts by two researchers who then had to try to reach agreement on study inclusion. Any discrepancies between researchers were resolved through consensus and, if necessary, by consulting a third reviewer. Full papers were obtained for studies that fitted the inclusion criteria, and each paper was reviewed for quality and data extracted. In addition to the computerized search, references from included studies were also checked (i.e. snowball method) to ensure that no relevant publications had been omitted.

\section{Data extraction and synthesis}

The following information was extracted: first author, year of publication, type of study, study location, workplace, intervention, characteristics of participants, outcome measurements, follow-up periods, and key findings.

The interventions were classified according to their outcomes that were defined in the following domains: 1) Policy for older workers, 2) Job retention, 3) Workability and productivity, 4) Health and well-being. Articles were included in one or more groups according to the main endpoints of the study. The framework described was developed by taking into account information obtained from "Healthy Work in an Ageing Europe", the $5^{\text {th }}$ Initiative of the European Network of Workplace Health Promotion [27] and other relevant milestones from gray literature [28].

\section{Quality assessment}

The methodological quality of each study was assessed using an Effective Public Health Practice Project (EPHPP) "Quality Assessment Tool for Quantitative Studies" that evaluates six main domains: (1) selection bias; (2) study design; (3) confounders; (4) blinding; (5) data collection method; and (6) withdrawals/dropouts, each of which can be rated as good, mediocre or poor. An overall score was obtained for each study by adding up the separate section rating (see Additional file 1).

The systematic review was conducted in accordance with the Preferred Reporting Items for Systematic Reviews and Meta-Analyses (PRISMA) guidelines.

\section{Results}

The literature search yielded 9791 papers, 3077 of which were duplicate studies. After initial review of the titles and abstracts, 6415 articles were excluded. Two reviewers independently examined 299 potentially eligible articles. 18 studies [14, 29-45] describing a wide range of promotion interventions were included in the final systematic review (Fig. 1). Five studies [32, 36-39] overlapped domains 3 and 4 . The characteristics of each eligible study are illustrated in Table 1.

The study populations came from different geographical sources: ten were European studies (five were conducted in the Netherlands, three in Finland, one in Germany and one in Sweden), five were North American (USA) studies, two were conducted in Australia and one in Taiwan.

Considerable variation was found both in the workplaces described and in the age used to define the "older" workers who participated in the studies. Most authors considered employees of 40/45 years to be older workers, although the mean age of study participants usually ranged from 51 to 55 years. The widest age range 


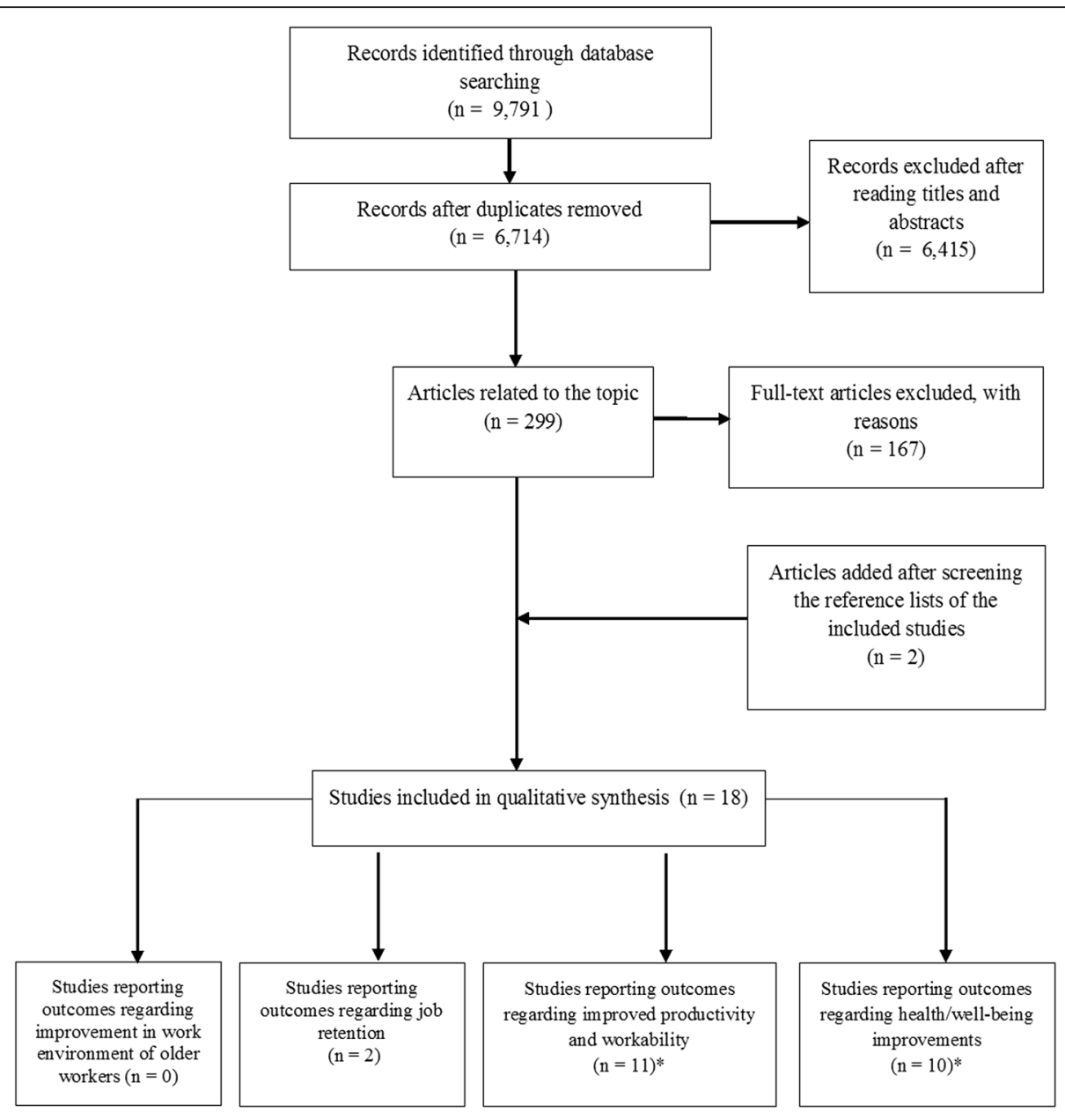

Fig. 1 Flow chart

* Five studies overlapping between groups

was reported by McDonald who referred to nurses between 40 and 70 years as mature age mentors.

As regards study design, eight studies were randomized controlled trials (RCTs), and four non randomized controlled trials (NCTs); there were also two cohort studies, one cross-sectional study, one pre-post study, one cost-effectiveness analysis, and one collective case study. Selected studies differed considerably in methods and type of intervention, outcomes and follow-up periods.

EPHPP analysis of interventions, based on study design, selection bias, randomization criteria, confounders, blinding, withdrawals and drop-outs, intervention integrity, and analyses revealed that 2 out of the 18 studies were of good overall quality, while 8 were mediocre and 5 were of poor quality (3 studies were not classifiable).

Domain 1-Policy for older workers Initially the review focused on identifying interventions that addressed the development of policies for OWs, aimed at improving interpersonal communication between the latter and other workplace employees, or combatted the exclusion or discrimination of OWs. Only a few [46-54] narrative studies were found to refer to the risk of ageism or express the need for a policy for OWs in the workplace, but since none of these described any intervention, they were not included in the review.

Domain 2-Increasing job retention

In the USA, Wallen and Mulloy [29] evaluated the response of a small sample of electronic company workers to a computer-based respiratory safetytraining program. Three versions of the program (text, text with pictures, text with pictures and audio narration) were shown to employees who then took a high- and a low-level learning test. Younger workers (under 44 years of age) did better overall. No significant effects of age or treatment were observed on low level learning, while workers over the age of 45 years improved in the high-level learning test 


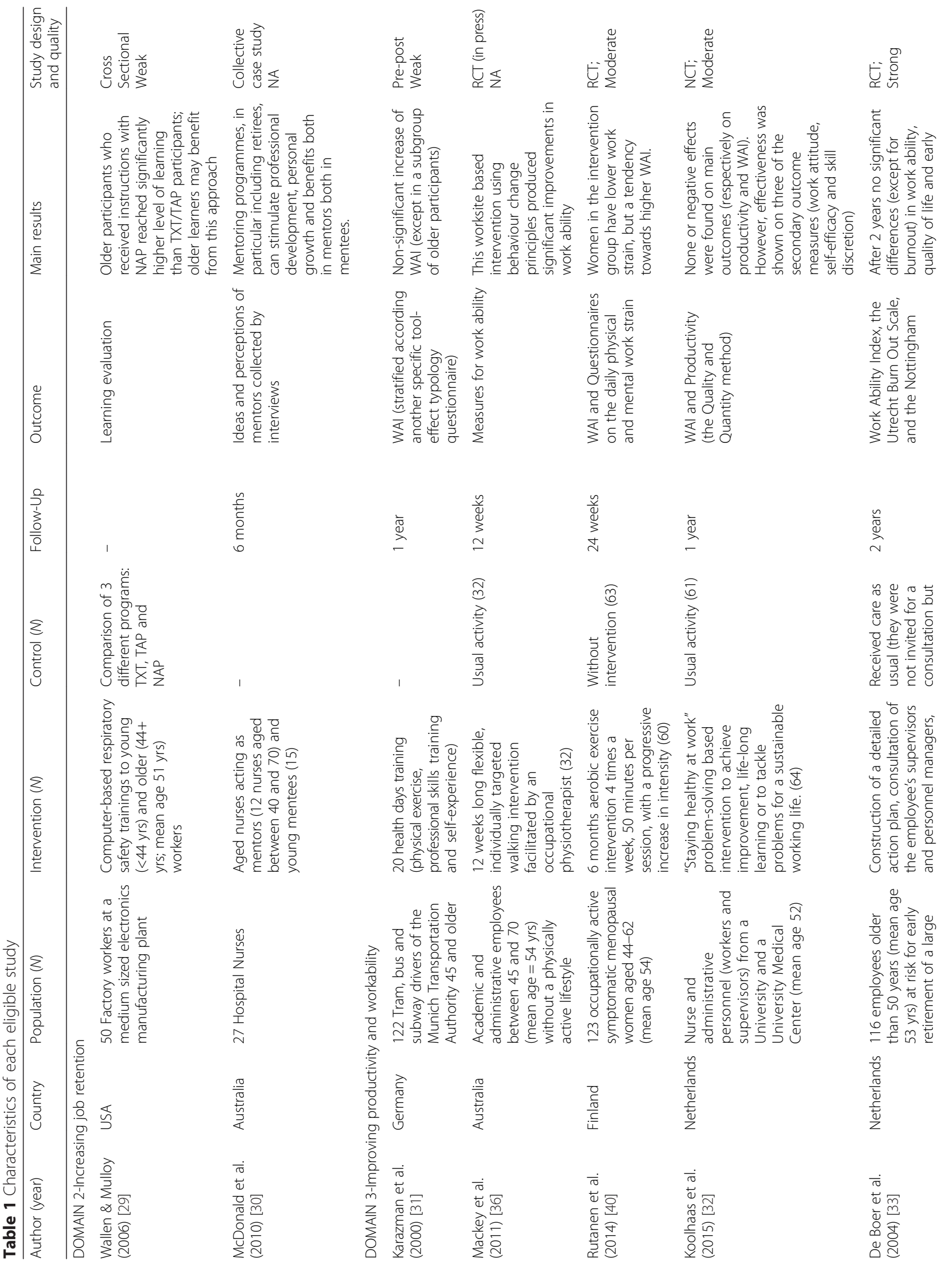




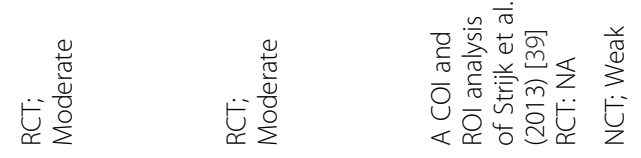

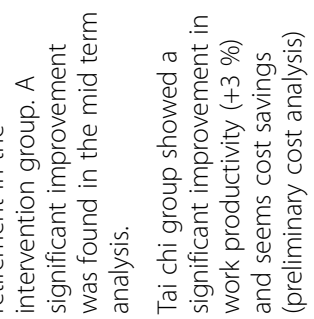

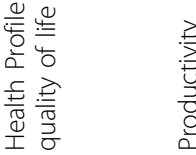

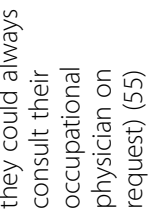

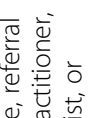

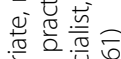

政

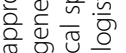

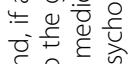

두요

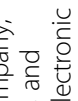

ํㅣㅇ 员

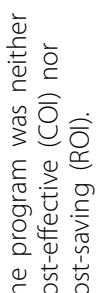

$\stackrel{\frac{\pi}{4}}{3}$

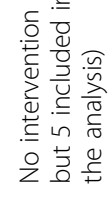

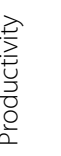

s.

Чั

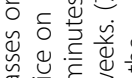
苋氞产岁声 능으은

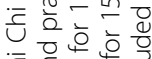

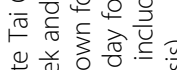

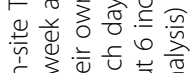

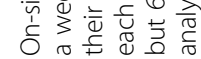

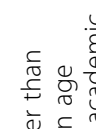

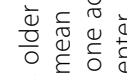

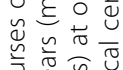

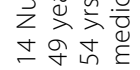

$\overleftrightarrow{s}$

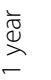

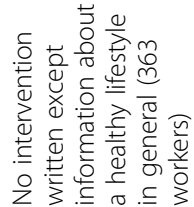

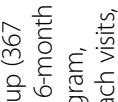

30

证

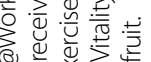

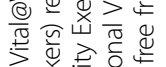

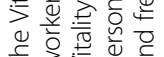

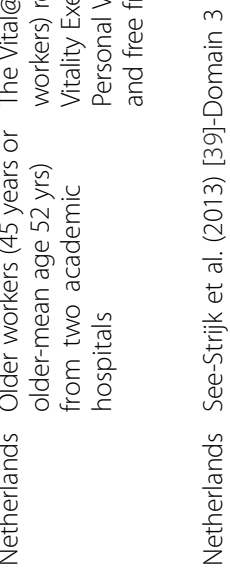

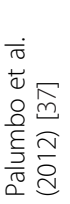

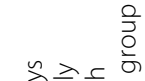

高势

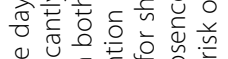

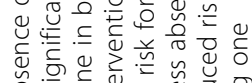

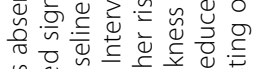

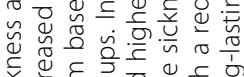

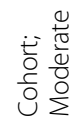

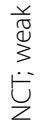

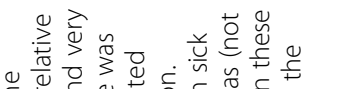

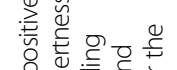

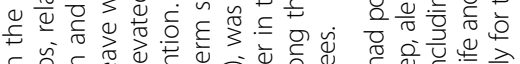

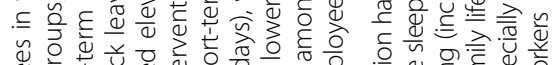

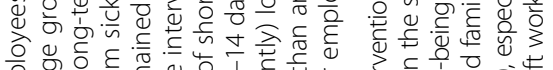
है

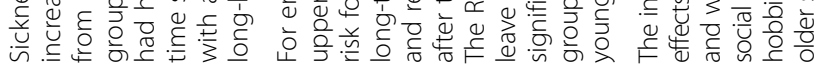

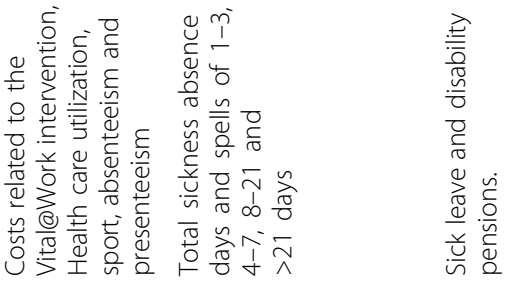

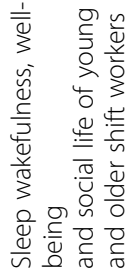

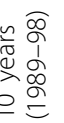

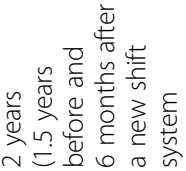

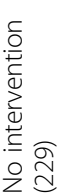

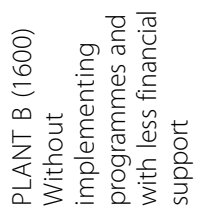

竞:

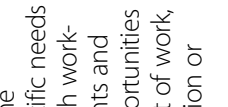

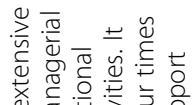

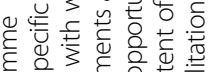

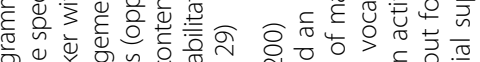

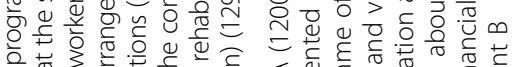

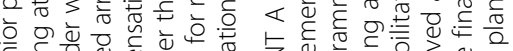

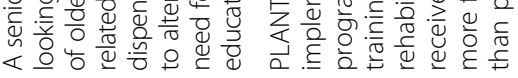

वृ.

产离离

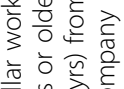

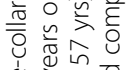

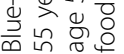

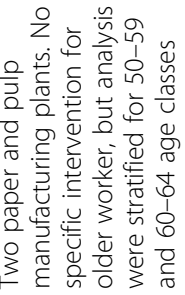

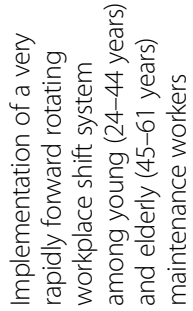

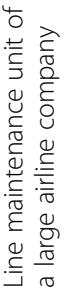

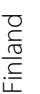

离

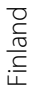

\&્d

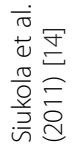

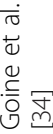

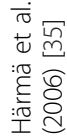


$\underset{\square}{\frac{0}{\frac{\pi}{0}}}$

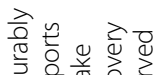

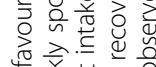

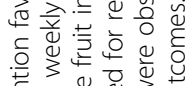

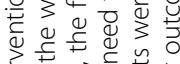

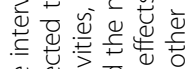

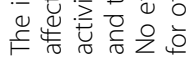

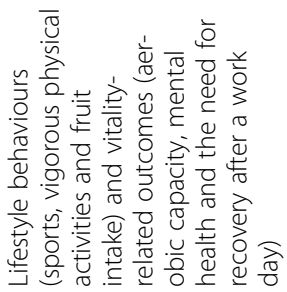

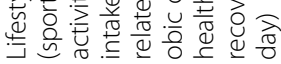

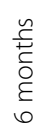

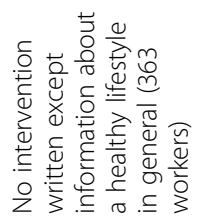

总突

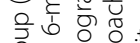
응중을

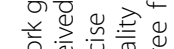

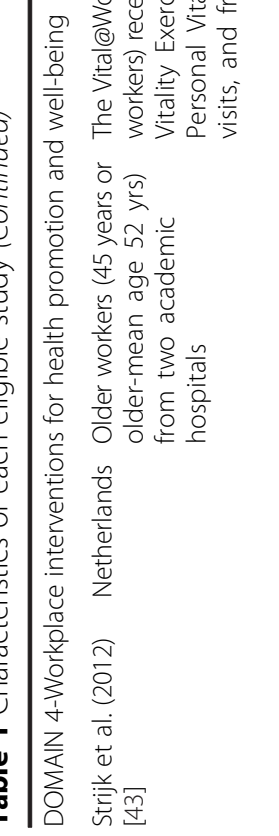

$\underset{\square}{\frac{\pi}{0}}$

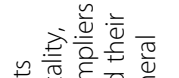

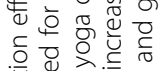

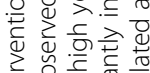

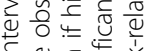

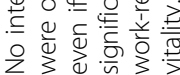

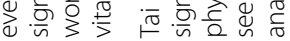

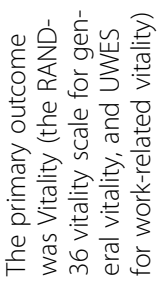

$\stackrel{\sqrt[\varpi]{ٍ}}{\beth}$

峁
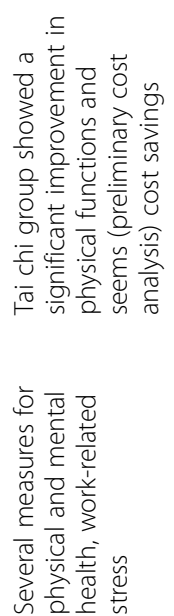

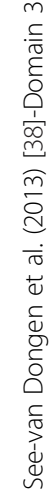

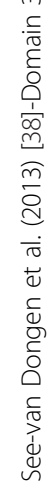

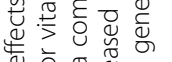

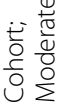
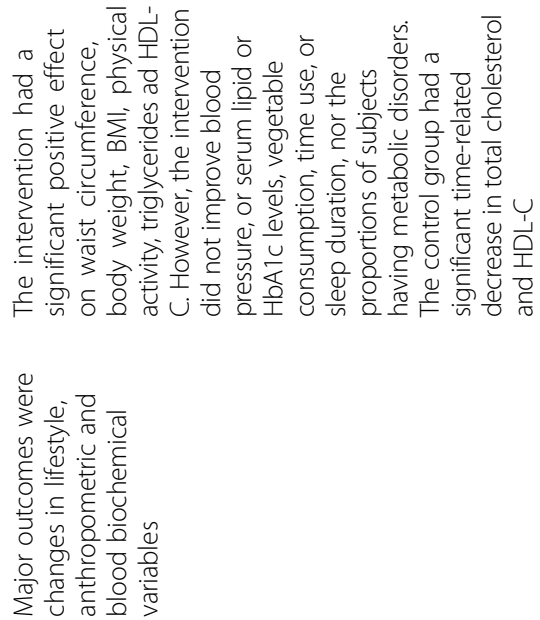

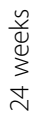

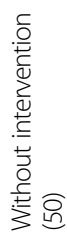

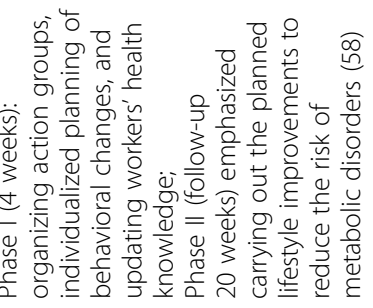

离离结

o

엉해

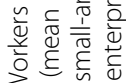

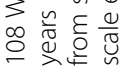

$\stackrel{凶}{\backsim}$

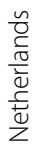

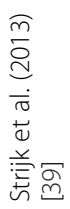

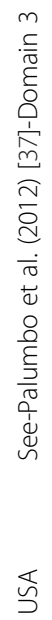

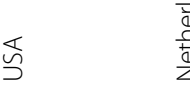

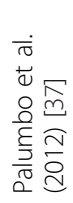

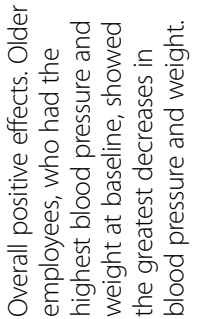

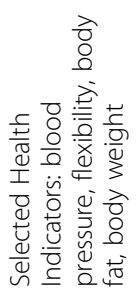

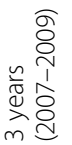

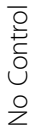

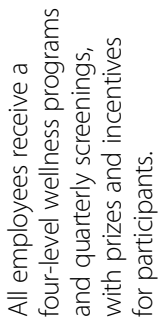

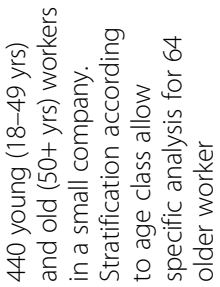

گ

흠

迹

产 


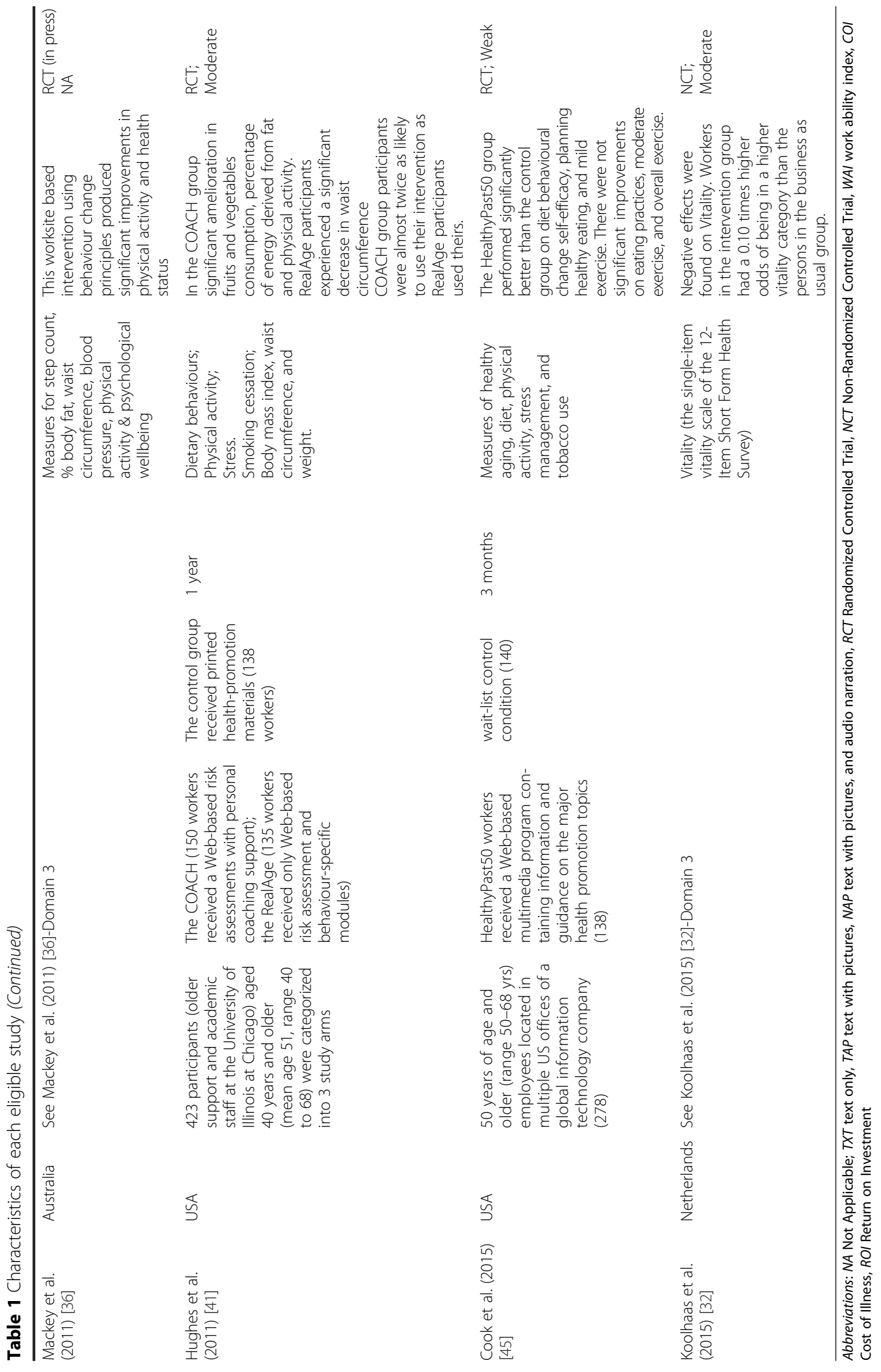


only after computer-based training with pictures and audio narration. McDonald et al. [30] proposed a mentoring service for nurses provided by a small group of older and retired nurses. Implementation of this program brought benefits for both mentors and mentees since it produced positive effects in three main areas by facilitating work and life decisions, by visibly helping other nurses and midwives, and by adapting to the role and the mentee. The mentors were a valuable source of knowledge; they also helped their mentees to manage and enjoy a long-term nursing career and cope with the high demands of the work environment. Moreover, they promoted the professional development and personal growth of mentees.

Domain 3-Improving productivity and workability 11 studies [14, 31-40] reported interventions aimed at improving work ability, work organization and productivity in OWs, and at postponing the prospect of early retirement. However, a real change in work organization was the main experimental intervention in only 5 of these studies [14, 32-35]; most of the studies explored the effect of various physical training programs on the aforementioned outcomes [31, 36-40].

3.1 Workplace interventions for maintaining work ability and postponing early retirement

Most studies [31-33, 36, 40] used the work ability index (WAI) as a way of self-assessing individual ability to deal with work demands. Karazman et al. [31] reported intervention in a subgroup of older participants selected through the "effect typology" questionnaire that identifies specific psychobiological patterns of response to intervention. Results from this study yielded a non-significant increase in the WAI after a 1-year health promotion program based on physical, psychological and stress management training accompanied by diet counselling. However, the authors highlighted a salutogenic effect of $\mathrm{OH}$ promotion intervention, due to an increase in the WAI and a decline in the desire for early retirement.

Mackey et al. [36] investigated the possible effects on workability of a 12-week individually targeted walking program. Preliminary results indicated that this kind of worksite based intervention, individually tailored for OWs, can produce significant improvements in physical activity and, it is hoped, in work ability.

Similarly, Rutanen et al. [40] investigated the effects on work ability and strain among menopausal female workers of a 24-week physical exercise program. At the end of this intervention, physical strain was lower in the treated group than in controls; however, differences in the WAI were not significant.

The study by Koolhaas et al. [32] assessed intervention aimed at creating a motivating and healthy work environment through the use of problem-solving techniques. The program failed to produce effects on productivity, but had a significant, negative effect on the WAI. Nevertheless, the program was shown to be effective with regard to some secondary outcomes such as work attitude, self-efficacy, and skill discretion.

In a very small self-selected group of OWs from an electronic company, intervention that included consultation with supervisors, the development of an action plan and referral to medical care when appropriate proved to be effective in reducing the frequency of early retirement after a short period (6 months). On the other hand, the overall rate of retirement (including disability retirement) in the intervention group was similar to controls at the end of the follow-up [33].

3.2 Workplace interventions for improving work organization and productivity

A limited number of studies reported interventions that focused on productivity, absenteeism, sickness absence and presenteeism in OWs.

A pilot study was conducted by Palumbo et al. [37] on a very small group of workers (6 nurses) to evaluate the feasibility of a Tai Chi workplace wellness program. Most of the results failed to show statistically significant group differences in changes over time.

In the Vital@Work study, a 6-month lifestyle intervention in the workplace that aimed to increase older workers' productivity and decrease sick leave by improving mental (e.g. via yoga sessions) and physical (e.g. via aerobic exercising) vitality factors, failed to show any significant differences between cases and controls [39]. Subsequent cost-benefit analysis [38] failed to reveal any significant positive findings related to absenteeism and presenteeism, thus indicating that this program was neither cost-effective (from a societal point of view) nor cost-saving (for employers).

In a Finnish food company, Siukola et al. [14] introduced a program for OWs designed to increase workability and the willingness to work until age-based retirement. A significant increase in the median number of sickness absence days per person/year was reported for the intervention and control groups during the follow-up period. Compared to the control group, the intervention 
group had a higher odds for short-term sickness absence, with a reduced odds of long-lasting sickness absence.

Only one study was designed to evaluate the effect on the personal and relational life of workers of an organizational intervention involving the implementation of a very rapid forward rotating workplace shift system [35]. The authors concluded that the ergonomic change had positive effects on sleep, alertness and well-being (including social and family life and hobbies), especially in the older shift workers.

Domain 4-Workplace interventions for health promotion and well-being

10 studies [32, 36-39, 41-45] reported interventions aimed at promoting health and lifestyle changes or reducing the risk of ill health in OWs.

The main outcome assessment made in the aforementioned Vital@Work study [43] concerned vitality. After the initial 6-months of follow up, the authors observed positive, statistically significant effects on sports activities and fruit intake, as well as on the need for recovery after a work day. However, no improvements were observed in vigorous physical activity, aerobic capacity or mental health. Furthermore, at 12-month follow up, no effects of intervention were observed for the main outcome, although there was a significant increase in the work-related and general vitality of the subgroup of high yoga compliers.

Similar, though non statistically significant results, were reported for the Tai Chi workplace wellness program in the aforementioned study of Palumbo et al. [37].

WHP programs were also implemented in small and medium-sized companies. A 24-week intervention trial designed to improve lifestyle, team spirit and goal keeping in workers aged 50+ years from small and medium scale enterprises in Taiwan significantly reduced workers' waist circumference, body weight and BMI [44]. However, this intervention did not improve blood pressure, serum lipid, or HbA1c (glycated haemoglobin) levels, vegetable consumption, time use, or sleep duration. Merrill et al. reported an experiment in medium-sized companies [42] that involved promoting physical activity, better nutrition, smoking cessation, and health education seminars. All workers were involved in quarterly monitoring. Retrospective analysis of 3 years of activity showed overall positive effects. Employees aged 50 or older, who had the highest blood pressure and weight at baseline, showed the greatest decrease in these parameters after the follow-up period.
Some interventions were based on specifically designed websites. A web-based multimedia program containing information and guidance on the major health promotion topics of healthy aging, diet, physical activity, stress management, and tobacco use, proved to be effective in obtaining behavioral modifications in mature aged workers [45]. The availability of personal coaching support in addition to web-based health risk assessment significantly increased the effectiveness of a WHP program [41].

\section{Discussion}

To the best of our knowledge, this is the first systematic review specifically devoted to investigating and assessing the quality level of studies regarding WHP programs for OWs This paper updates the evidence provided by previously published reviews about health promotion and well-being in OWs [18-20], but also includes other relevant domains of a comprehensive WHP approach (job retention and productivity/workability). Our study shows that, although this subject is of great importance, there are only a few studies that evaluate WHP actions for OWs. The literature on recent interventions to improve the working climate, train older workers, provide ergonomic conditions at the workplace and promote or maintain health in the aging workforce is rather limited. The WHP programs are aiming different areas: physical exercise, eating habits, walking, etc. It may be that different programs need different time to show an effect. For example: people may get fitter much faster with exercise program than changing their beliefs. Unfortunately, most of the available studies have been conducted on small samples, for a limited period of time, with methods the same author sometimes described as unsatisfactory. Evidence of effectiveness is often lacking, especially in the long term. The considerable variability in methods, standards and outputs, in addition to the overall mediocre quality of most of the studies, make it difficult to draw conclusions.

Studies on the training of older workers have usually been conducted on very small convenience samples, making it difficult to generalize results. However, the finding that learning in OWs differs from that in younger employees, and that it benefits from a multimodal approach [29] is in agreement with the general orientation of the literature [55]. Likewise, there is no reason to believe that the experience described by McDonald et al. [30], in which OWs were used as mentors, cannot be repeated successfully in other companies. As the authors claim, by respecting and enhancing diverse points of views and experiences, mentoring initiatives offer an important intergenerational and cross-cultural opportunity 
to facilitate the creation of a learning environment and trusting relationships.

An analysis of the actions that have attempted to change work ability, absenteeism, or retirement intentions, inevitably reveals a discrepancy between resources and objectives. WHP interventions based on short-term physical exercise generally failed to improve the work ability of workers [31, 32, 40], or at best obtained a transient improvement that soon disappeared [33]. The observation period was usually too short to ascertain whether these programs were able to at least prolong previous ability. Similar conclusions can be reached for the studies that aimed at reducing early retirement [31], absenteeism or presenteeism $[37,38]$. In some cases the observed effect was of dubious benefit [14], since it produced only a shift in the pattern of absences, not a reduction in absenteeism. The authors' statement, that the increase in short-term absence (so-called "compensatory absence") can be linked to a reduction in early retirement, is controversial. In fact previous studies have demonstrated that workers who make use of short-term absences, have a high level of selfperceived job strain and a low level of social support at work [56]. Although in the planning and pilot phases of the studies the attitude of researchers was always optimistic about the possibility of significantly improving the health and productivity of OWs [36, 37], after the follow-up period, results were often questionable, or even negative [38]. On examining these findings we cannot definitively rule out the possibility that paradoxal results, i.e. the worst effects seen in the intervention group, could be due to the small number of observations or unsatisfactory epidemiological methods. Even in our search we came across a large number of experiences and recent reviews on this issue [20], we confirmed a lack of longitudinal research and studies of high quality. Additional intervention studies are needed to support evidencebased decision for ageing workers.

Programs aimed at maintaining the health and general well-being of OWs varied greatly across studies and seemed to be much more effective than those focused on changing workplace conditions or improving work ability and productivity. The most common interventions included physical activity training, such as aerobic exercises, yoga or Tai Chi courses, walking programs, etc. We must confirm, however, that there is still a large number of research gaps, including the lack of longitudinal studies on some relevant disorders, such as stress and anxiety, musculoskeletal disorders, accidents, and suicide risk in OWs, as previously observed [18].

In general, we may observe thatWHP is thriving in the USA, while European studies are limited to the experiences of a few research groups, mainly in Northern countries. One of the reasons for this is that in many European countries the cost of employee sickness absence is generally incurred by the NHS (National Health Service), and this does not encourage companies to spend on health prevention [57], or provide information for educative purposes and offer self-help programs. Another important factor is that the health and safety of workers traditionally follows a labor approach: prevention only applies to aspects related to work, while general health protection and promotion (e.g. health related to life-style) are not included in this framework [58]. Moreover, as the increase in the age limit for retirement is a very recent innovation in all European countries, there has not been enough time to prepare and implement promotion programs specifically targeted at workers over 55 years of age. Finally, it is clear that, from the point of view of companies that intend to promote the health of their workers, intervention should not be limited to older workers alone, but should ensure that there is a beneficial effect for all employees. Employers who provide WHP programs make an investment in human capital; they rightly expect to have a return over time, not only in terms of health, but also in productivity and product quality as shown in previous studies on the whole workforce [59]. The sensitivity of companies towards health promotion does not depend on size, total income or other econometric parameters, but mainly on corporate culture. Workplace actions for health are often supported by medium-sized or small companies, with some studies showing the best results in the older workforce [42].

The workplace is an ideal setting for implementing health promotion activities [60-62] because there is greater access to workers in a controlled environment through existing channels of communication and social support networks, e.g. $\mathrm{OH}$ Services that facilitate the creation of a supportive culture [63]. The worksite is also a cost-effective setting for providing health education and promoting health behavior change [64, 65]. However, our research confirms the observation of McDermott et al. [20] that very few $\mathrm{OH}$ interventions have addressed the health and workability of OWs. There is a wide space to improve $\mathrm{OH}$ commitment and account the needs of the older workforce. Our review confirms that WHP interventions are seen as positive by older workers but it is important to ensure equal access to all workers in such promotions, as previously suggested [18].

Our study has some limitations. The heterogeneity of the studies we have included makes it difficult to perform a synthesis of the literature, and the low quality of most of the studies weakens the evidence obtained. Interventions conducted on small convenience samples cannot be applied to the entire working population. Moreover, as in the majority of reviews, publication bias might be an issue. 
In conclusion, the insufficient and limited evidence available for a favourable effect of WHP programs is mainly due to a scarcity of RCTs and inconsistent findings between the limited number of studies. The aging of the workforce is a very recent phenomenon and this has so far not allowed the publication of prospective studies of high quality.

Although there seems to be a number of studies that report WHP interventions, especially in the grey literature, the results of the present review emphasize the need for high-quality studies, with follow-up periods that would help researchers to ascertain the effects of intervention and complement them by performing cost/ benefit analyses. Besides measuring cost effectiveness of WHP interventions for individuals and their economic impact on the companies, ethical aspects should also be taken into account in the evaluation process. At the moment, the paucity of studies on WHP for OWs prevents a detailed analysis of the relevant bioethical and social issues connected to the problem. In a resource-limited and employment-limited system, making efforts to improve the employability of OWs undoubtedly might decrease the employment of younger ones. In our opinion, rather than thinking about improving the conditions of an age category, we should improve the working conditions of all. Improving health, safety and welfare of workers is a benefit for the whole society. We think this topic may be a good argument for future research.

\section{Conclusions}

On the whole, the evidence currently available does not unequivocally support the effectiveness of WHP interventions targeted at OWs in reducing sickness absence, presenteeism, or the intention to retire in this population. Also there is insufficient evidence that worksite programs can increase the working capacity of older employees. However, there is moderate evidence that active workplace intervention reduces waist circumference, body weight, BMI and other components of metabolic syndrome, and limited evidence that web-based programs may be useful in changing worker's behavior. Older workers' health can therefore be improved with active workplace interventions, but careful consideration must be given to the content, quality and cost-effectiveness of this type of intervention.

\section{Additional files}

Additional file 1: Quality assessment of the included studies. (XLSX 17.0 KB)

Additional file 2: PRISMA statement for reporting systematic reviews. (DOCX 30.4 KB)

\section{Abbreviations}

BMI, body mass index; EPHPP, Quality Assessment Tool for Quantitative Studies; $\mathrm{HbA} 1 \mathrm{c}$, glycated haemoglobin; NCTs, non randomized controlled trials; $\mathrm{OH}$, occupational health; OW, older worker; PRISMA, Preferred Reporting Items for
Systematic Reviews and Meta-Analyses; RCTs, randomized controlled trials; VO2 max, maximal oxygen consumption, maximal oxygen uptake, peak oxygen uptake or maximal aerobic capacity; WAI, work ability index; WHP, workplace health promotion.

\section{Acknowledgements \\ This publication arises from the project Pro-Health $65+$ which has received funding from the European Union, in the framework of the Health Programme (2008-2013). The content of this publication represents the views of the authors and it is their sole responsibility; it can in no way be taken to reflect the views of the European Commission and/or the Executive Agency for Health and Consumers or any other body of the European Union. The European Commission and/or the Executive Agency do(es) not accept responsibility for any use that may be made of the information it contains. The Authors thank Ms. Elisabeth Ann Wright for assistance with the English version of this manuscript.}

\section{Declarations}

This article has been published as part of BMC Health Services Research Volume 16 Supplement 5, 2016: Economic and institutional perspectives on health promotion activities for older persons. The full contents of the supplement are available online at http://bmchealthservres.biomedcentral.com/articles/ supplements/volume-16-supplement-5.

\section{Funding}

This publication arises from the project Pro-Health $65+$ which has received funding from the European Union, in the framework of the Health Programme (2008-2013). The content of this publication represents the views of the authors and it is their sole responsibility; it can in no way be taken to reflect the views of the European Commission and/or the Executive Agency for Health and Consumers or any other body of the European Union. The European Commission and/or the Executive Agency do(es) not accept responsibility for any use that may be made of the information it contains.

Availability of data and materials

The systematic review was conducted in accordance with the Preferred Reporting Items for Systematic Reviews and Meta-Analyses (PRISMA) guidelines (included as Additional file 2).

\section{Authors' contributions}

AP and NM conceived the design of the study, drafted the manuscript and critically revised it; SM and JS performed the acquisition and analysis of data and the quality assessment; DLM, AC and AB collaborated in data acquisition, analysis and interpretation; UM and WR critically revised the manuscript. All authors read and approved the final manuscript.

\section{Authors' information}

WR is President of the Italian Institute of Health (ISS). UM is Associate Professor, NM is Aggregate Professor, AP and AC are Researchers at the Università Cattolica del Sacro Cuore, Rome. SM and JS are PhD at the Università Cattolica del Sacro Cuore, Rome. DLM and AB are resident doctors at the Università Cattolica del Sacro Cuore, Rome.

\section{Competing interests}

The authors state that they have no competing interest in this field.

\section{Consent for publication}

Not applicable. This is a literature review.

Ethics approval and consent to participate

Not applicable. This is a literature review.

\section{Author details}

${ }^{1}$ Department of Public Health, Università Cattolica del Sacro Cuore, Rome, Italy. ${ }^{2}$ Department of Gerontology, Orthopedics and Neuroscience, Università

Cattolica del Sacro Cuore, Rome, Italy. ${ }^{3}$ Istituto Superiore di Sanità, Rome, Italy.

Published: 5 September 2016 


\section{References}

1. Granville G, Evandrou M. Older men, work and health. Occup Med (Chic III). 2010;60(3):178-83.

2. European Commission - Directorate-General for Economic and Financial Affairs. The 2015 Ageing Report. Underlying Assumptions and Projection Methodologies. EUROPEAN ECONOMY 8-2014. http://ec.europa.eu/ economy_finance/publications/european_economy/2014/pdf/ee8_en.pdf. Accessed 12July 2016.

3. IImarinen J. Aging workers. Occup Environ Med. 2001;58(8):546-52.

4. Delloiacono N. Musculoskeletal safety for older adults in the workplace. Workplace Health Saf. 2015;63(2):48-53

5. Loeppke RR, Schill AL, Chosewood LC, Grosch JW, Allweiss P, Burton WN. Advancing workplace health protection and promotion for an aging workforce. J Occup Environ Med. 2013;55(5):500-6.

6. CDC National Center for Chronic Disease Prevention and Health Promotion. Older Employees in the Workplace. Issue Brief No. 1, July 2012. http://www. public-health.uiowa.edu/hwce/wp-content/uploads/2014/11/Issue_Brief_ No_1_Older_Employees_in_the_Workplace_7-12-2012_FINAL508.pdf. Accessed 15 September 2015.

7. The U.S. Equal Employment Opportunity Commission (EEOC). The Age Discrimination in Employment Act. Pub. L. No. 90-202. https://www.eeoc. gov/laws/types/age.cfm. Accessed 12 July 2016.

8. Brooke E, Goodall J, Handrus M, Mawren D. Applying workability in the Australian residential aged care context. Australas J Ageing. 2013;32(2):130-4.

9. Ross D. Ageing and work: an overview. Occup Med (Lond). 2010;60(3):169-71.

10. Bonomini F, Rodella LF, Rezzani R. Metabolic syndrome, aging and involvement of oxidative stress. Aging Dis. 2015;6(2):109-20.

11. Abraha I, Cruz-Jentoft A, Soiza RL, O'Mahony D, Cherubini A. Evidence of and recommendations for non-pharmacological interventions for common geriatric conditions: the SENATOR-ONTOP systematic review protocol,". BMJ Open. 2015; vol. 5, no. 1.

12. Knoche K, Sochert R, Houston K. Promoting healthy work for workers with chronic illness: A guide to good practice. European Network for Workplace Health Promotion (ENWHP), 2012. http://www.enwhp.org/uploads/media/ ENWHP_Guide_PH_Work_final.pdf. Accessed 12 July 2016.

13. Furunes T, Mykletun RJ. Age discrimination in the workplace: validation of the Nordic Age discrimination scale (NADS). Scand J Psychol. 2010;51(1):23-30.

14. Siukola A, Virtanen $\mathrm{P}$, Huhtala $\mathrm{H}$, Nygård $\mathrm{CH}$. Absenteeism following a workplace intervention for older food industry workers. Occup Med (Chic III). 2011;61(8):583-5.

15. Greco E, Osnato OU, Magnavita N. Fabbisogni formativi per l'esercizio del ruolo di Medico Competente. IAS Istituto per gli Affari Sociali, Roma, 2015. http://www.safersrl.it/risorse/100528_IAS_fabbisogni_formativi_MC.pdf. Accessed 12 July 2016.

16. Magnavita N. Engagement in health and safety at the workplace: a new role for the occupational health physician. In: Graffigna G. (eds) "Promoting Patient Engagement and Participation for Effective Healthcare Reform" IGI Global, Hershey, Pennsylvania, 2016.

17. Maes L, Van Cauwenberghe E, Van Lippevelde W, Spittaels H, De Pauw E, Oppert $J-M$, et al. Effectiveness of workplace interventions in Europe promoting healthy eating: a systematic review. Eur J Public Health. 2012;22(5):677-83.

18. Cancelliere C, Cassidy JD, Ammendolia C, Côté P. "Are workplace health promotion programs effective at improving presenteeism in workers? a systematic review and best evidence synthesis of the literature.,". BMC Public Health. 2011;11(1):395.

19. Crawford JO, Graveling RA, Cowie HA, Dixon K.The health safety and health promotion needs of older workers. Occup Med (Lond). 2010; 60(3):184-92. doi:10.1093/occmed/kqq028.

20. McDermott HJ, Kazi A, Munir F, Haslam C. Developing occupational health services for active age management. Occup Med (Lond). 2010;60(3):193-204. doi:10.1093/occmed/kqq026.

21. Cloostermans L, Bekkers MB, Uiters E, Proper KI.The effectiveness of interventions for ageing workers on (early) retirement, work ability and productivity: a systematic review. Int Arch Occup Environ Health. 2015 ;88(5):521-32. doi:10.1007/s00420-014-0969-y.

22. Pitt-Catsouphes M, James JB, Matz-Costa C. Workplace-based health and wellness programs: the intersection of aging, work, and health. Gerontologist. 2015;55(2):262-70.

23. Wegman D.H, McGee J.P. Health and Safety Needs of Older Worker: Report by Committee on the Health and Safety Needs of Older Workers. 2004. Washington, D.C.: The National Academies Press; doi:10.17226/10884.
24. Dordoni P, Argentero P. When Age stereotypes are employment barriers: a conceptual analysis and a literature review on older workers stereotypes. Ageing Int. 2015;40(4):393-412.

25. Ilmarinen J. The ageing workforce-challenges for occupational health. Occup Med (Lond). 2006;56(6):362-4.

26. Hymel PA, Loeppke RR, Baase CM, Burton WN, Hartenbaum NP, Hudson TW, McLellan RK, Mueller KL, Roberts MA, Yarborough CM, Konicki DL, Larson PW. Workplace health protection and promotion: a new pathway for a healthier-and safer-workforce. J Occup Environ Med. 2011;53(6):695-702. doi:10.1097/JOM.0b013e31822005d0.

27. Morschhäuser M, Sochert R. Healthy Work in an Ageing Europe. Strategies and Instruments for Prolonging Working Life. ENWHP European Network for Workplace Health Promotion. Essen, 2006. Accessible at: http://www. ageingatwork.eu/resources/health-work-in-an-ageing-europe-enwhp-3.pdf

28. Sitko SJ, Kowalska- Bobko I, Mokrzycka A, Zabdyr-Jamróz M, Domagała A, Magnavita N, Poscia A, Rogala M, Szetela A, Golinowska S. Institutional analysis of health promotion for older people in Europe. Concept and research tool. BMC Health Service Research, 2016 (in press).

29. Wallen ES, Mulloy KB. Computer-based training for safety : comparing methods with older and younger workers. J Safety Res. 2006;37:461-7.

30. McDonald G1, Mohan S, Jackson D, Vickers MH, Wilkes L. Continuing connections: the experiences of retired and senior working nurse mentors. J Clin Nurs. 2010;19(23-24):3547-54. doi:10.1111/j.1365-2702.2010.03365.x.

31. Karazman R, Kloimüller I, Geissler H, Karazman-Morawetz I. Effects of ergonomic and health training on work interest, work ability and health in elderly public urban transport drivers. Int J Ind Ergon. 2000;25(5):503-11.

32. Koolhaas W, Groothoff JW, de Boer MR, van der Klink JJ, Brouwer S. Effectiveness of a problem-solving based intervention to prolong the working life of ageing workers. BMC Public Health. 2015;15:76. doi:10.1186/ s12889-015-1410-5.

33. de Boer AG, van Beek JC, Durinck J, Verbeek JH, van Dijk FJ. An occupational health intervention programme for workers at risk for early retirement; a randomised controlled trial. Occup Environ Med. $2004 ; 61(11): 924-9$.

34. Goine H, Knutsson A, Marklund S, Karlsson B. Sickness absence and early retirement at two workplaces - effects of organisational intervention in Sweden. Soc Sci Med. 2004;58(1):99-108.

35. Härmä M, Tarja H, Irja K, Mikael S, Jussi V. A controlled intervention study on the effects of a very rapidly forward rotating shift system on sleep-wakefulness and well-being among young and elderly shift workers. Int J Psychophysiol. 2006;59:70-9.

36. Mackey MG, Bohle P, Taylor P, Di Biase T, Mcloughlin C, Purnell K. Walking to wellness in an ageing sedentary university community: design, method and protocol. Contemp Clin Trials. 2011;32(2):273-9.

37. Palumbo MV, Wu G, Shaner-Mcrae H, Rambur B, Mcintosh B. Tai Chi for older nurses : a workplace wellness pilot study. Appl Nurs Res. 2012;25(1):54-9.

38. van Dongen JM1, Strijk JE, Proper Kl, van Wier MF, van Mechelen W, van Tulder MW, van der Beek AJ. A cost-effectiveness and return-on-investment analysis of a worksite vitality intervention among older hospital workers: results of a randomized controlled trial. J Occup Environ Med. 2013 Mar 55(3):337-46. doi:10.1097/JOM.0b013e31827b738e.

39. Strijk JE, Proper Kl, van Mechelen W, van der Beek AJ. Effectiveness of a worksite lifestyle intervention on vitality, work engagement, productivity, and sick leave: results of a randomized controlled trial. Scand J Work Environ Health. 2013;39(1):66-75.

40. Rutanen R, Nygård C, Moilanen J, Mikkola T, Raitanen J. Effect of physical exercise on work ability and daily strain in symptomatic menopausal women : a randomized controlled trial. Work. 2014;47:281-6.

41. Hughes SL, Seymour RB, Campbell RT, Shaw JW, Fabiyi C, Sokas R. Comparison of two health-promotion programs for older workers. Am J Public Health. 2011;101(5):883-90.

42. Merrill R.M, Aldana S.G, Tonya P, Howe G, Anderson DR, Whitmer R.W. The Impact of Worksite Wellness in a Small Business Setting. J Occup Environ Med. 2011;53(2):127-31. doi:10.1097/JOM.0b013e318209e18b.

43. Strijk JE, Proper Kl, van der Beek a J, van Mechelen W. A worksite vitality intervention to improve older workers' lifestyle and vitality-related outcomes: results of a randomised controlled trial, J Epidemiol Community Heal. 2012;66(11):1071-8.

44. Chen M-M, Tsai a C, Wang J-Y. The effectiveness and barriers of implementing a workplace health promotion program to improve metabolic disorders in older workers in Taiwan, Glob Health Promot. 2014;0:1-9. 
45. Cook RF, Hersch RK, Schlossberg D, Leaf SL. A Web-based health promotion program for older workers: randomized controlled trial, J Med Internet Res. 2015;17(3):e82.

46. Brooke E, Taylor P, Mcloughlin C, Di Biase T. Managing the working body: active ageing and limits to the 'flexible' firm,. Ageing and Society. 2013;33(8): 1295-1314. doi:10.1017/S0144686X12000426.

47. Fogg NP, Harrington PE, Mcmahon BT. The triumph of older workers during the great recession: implications for employers and disability policy. J Vocat Rehabil. 2012;36(3):141-7.

48. Gabrielle S, Jackson D, Mannix J. Adjusting to personal and organisational change: views and experiences of female nurses aged $40-60$ years. Collegian. 2008;15(3):85-91.

49. Longo J. Bullying and the older nurse. J Nurs Manag. 2013;21:950-5.

50. Malinen S, Johnston L. Workplace ageism: discovering hidden bias. Exp Aging Res. 2013:39(4):445-65.

51. Melillo Devereaux K. Cognitive health and older workers. J Gerontol Nurs. 2013;39(6):13-8.

52. Piekkola H. Nordic policies on active ageing in the labour market and some European comparisons. Int Soc Sci J. 2006;58(190):545-57.

53. Thorsen S, Rugulies R, Løngaard K, Borg V, Thielen K, Bjorner JB. The association between psychosocial work environment, attitudes towards older workers (ageism) and planned retirement. Int Arch Occup Environ Health. 2012;85(4):437-45.

54. Truxillo D.M, Cadiz D.M, Hammer L.B. Supporting the Aging Workforce: A Review and Recommendations for Workplace Intervention Research. Annual Review of Organizational Psychology and Organizational Behavior. 2015;2: 351-81. doi:10.1146/annurev-orgpsych-032414-111435.

55. Decarli C, Kawas C, Morrison JH, Reuter-Lorenz PA, Sperling RA, Wright CB. Session II: mechanisms of age-related cognitive change and targets for intervention: neural circuits, networks, and plasticity. J Gerontol A Biol Sci Med Sci. 2012;67(7):747-53.

56. Magnavita N, Garbarino S. Is absence related to work stress? a repeated crosssectional study on a special police force. Am J Ind Med. 2013;56(7):765-75.

57. Downey AM, Sharp DJ. Why do managers allocate resources to workplace health promotion programmes in countries with national health coverage? Health Promot Int. 2007;22(2):102-11.

58. Vanhoorne M.N, Vanachter O.V, De Ridder M.P, Occupational health care for the 21 st century: from health at work to workers' health. Int. J. Occup. Environ. Health. 2006;12(3):278-85.

59. Parks K.M, Steelman L.A. Organizational wellness programs: A metaanalysis, J Occup Health Psychol. 2008;13(1):58-68. doi:10.1037/1076-8998.13.1.58.

60. Pegus C, Bazzarre TL, Brown JS, Menzin J. Effect of the heart at work program on awareness of risk factors, self-efficacy, and health behaviors. J Occup Environ Med. 2002:44(3):228-36.

61. Aldana SG, Greenlaw R, Diehl HA, Englert H, Jackson R. Impact of the coronary health improvement project (CHIP) on several employee populations. J Occup Environ Med. 2002;44(9):831-9.

62. Bloch MJ, Armstrong DS, Dettling L, Hardy A, Caterino K, Barrie S. Partners in lowering cholesterol: comparison of a multidisciplinary educational program, monetary incentives, or usual care in the treatment of dyslipidemia identified among employees. J Occup Environ Med. 2006;48(7):675-81.

63. CDC National Center for Chronic Disease Prevention and Health Promotion. Public Health Strategies for Preventing and Controlling Overweight and Obesity in School and Worksite Settings. A Report on Recommendations of the Task Force on Community Preventive Services. MMWR Morbidity and Mortality Weekly Report 2005; 54: RR-10. Accessible at: http://www.cdc.gov/ $\mathrm{mmwr} / \mathrm{pdf} / \mathrm{rr} / \mathrm{rr} 5410 . \mathrm{pdf}$

64. Chapman L. S. Expert opinions on 'best practices' in worksite health promotion (WHP). Am J Health Promot. 2004:18(6):1-6.

65. Magnavita N SA, De Lorenzo G. Health promotion in the workplace. Med Lav. 2014:105(6):473-4.

\section{Submit your next manuscript to BioMed Central and we will help you at every step:}

- We accept pre-submission inquiries

- Our selector tool helps you to find the most relevant journal

- We provide round the clock customer support

- Convenient online submission

- Thorough peer review

- Inclusion in PubMed and all major indexing services

- Maximum visibility for your research

Submit your manuscript at www.biomedcentral.com/submit
Biomed Central 